\title{
Non-adiabatic perturbations in multi-component perfect fluids.
}

\author{
N.A. Koshelev ${ }^{*}$ \\ Ulyanovsk State University, Leo Tolstoy str 42, 432970, Russia
}

(Dated: December 28, 2017)

\begin{abstract}
The evolution of non-adiabatic perturbations in models with multiple coupled perfect fluids with non-adiabatic sound speed is considered. Instead of splitting the entropy perturbation into relative and intrinsic parts, we introduce a set of symmetric quantities, which also govern the non-adiabatic pressure perturbation in models with energy transfer. We write the gauge invariant equations for the variables that determine on a large scale the non-adiabatic pressure perturbation and the rate of changes of the comoving curvature perturbation. The analysis of evolution of the non-adiabatic pressure perturbation has been made for several particular models.
\end{abstract}

PACS numbers: $98.80 . \mathrm{Cq}$

\section{INTRODUCTION.}

Multi-component models play an important role in modern cosmology. They are required to study a large scale structure formation [1, 2] and are used in multi-field inflationary models [3 [5]. Multi-fluid models are also essential in the context of preheating [6, 7]. It is well known that in cosmological models with multiple fractions the growing mode solution of the curvature perturbation on comoving hypersurfaces $\mathcal{R}$ remains constant on a large scale in the absence of any entropy perturbations [1, 2, [8]. This quantity is valuable to cosmological applications, because, for example, it allows one to relate the perturbations generated at a stage of inflation with the primordial perturbations in the early radiation dominated era. In the presence of the non-adiabatic pressure perturbations the comoving curvature perturbation $\mathcal{R}$ always changes with time. Thus, the non-adiabatic perturbations may affect the observable power spectrum of the adiabatic perturbations.

A scalar field and a cosmological fluid seem very different, but the distinctions are not fundamental. Any barotropic perfect fluid can be represented as a scalar field with a nontrivial kinetic part [9]. On the other hand, in the linear perturbation theory the scalar field with a self-coupling potential can be treated as a perfect fluid with sound speed $c_{s}^{2} \equiv \delta P / \delta \rho$ (computed in the fluid rest frame) different from adiabatic sound speed $c_{s(a d)}^{2}[10]$. For this reason, an effective fluid description is often used. Under this approach, a scalar field can be fully described by specifying its sound speed and the equation of state, and by phenomenologically introducing an energy-momentum transfer between the scalar field and other fluids. In slightly different and more general formalism, any system of $N$ coupled canonical scalar fields can be modelled by $N$ "kinetic" fluids with a stiff equation of state interacting with one "potential" fluid with a vacuum equation of state [1].

A valuable class of multi-component models is formed by the systems containing the coupled scalar fields and the barotropic fluids. These are, among others, the cosmological models with coupled cold dark matter and dark energy, considered as a canonical scalar field usually called quintessence. For these models there has been found the possibility of existence of large scale instabilities at the radiation dominated stage, caused by the fast growth of non-adiabatic perturbations [12]. Such early time instabilities have received much attention in a variety of scenarios [13 15].

Although non-adiabatic perturbations were investigated long ago, there still remain some unresolved issues. Currently, the definitions of the relative entropy perturbation for scalar fields [16, 17] (note that the authors of Refs. [18, 19] used adiabatic and entropy field combinations instead) and of general perfect fluids with intrinsic entropy perturbations [11, 20] are different. Thought Ref. [11] gives a unified definition of relative entropy that is appropriate both for barotropic fluids and for scalar fields, its use leads to some theoretical difficulties. In particular, even in the simplest single field models this method assumes, in general, a nonzero relative entropy between the "kinetic" and "potential" fluids, which seems somewhat artificial. In the phenomenological approach there are also some problems with the description of the interaction of these auxiliary fluids and others. It is also desirable to study the adiabatic condition in more detail. For example, in the recent paper [21] an analytical attractor solution for dark energy perturbations in the synchronous gauge at a constant dark energy equation of state parameter was obtained. For this solution, the usually imposed generalized adiabatic condition [18, 19] does not take place.

*Electronic address: koshna71@inbox.ru 
The aim of this work is to, at least partially, fill the gaps. The paper is organized as follows. In Section II we briefly review the perturbed Einstein and continuity equations for a general multi-fluid model with energy exchange. Assuming that the total energy density can be represented as a sum of energy densities of some perfect fluids, we write, in Section III, an evolution equation for the comoving curvature perturbation $\mathcal{R}$ and an expression for the non-adiabatic pressure perturbation $P_{n a d}$. Instead of using of relative entropy perturbations, we introduce a set of symmetric quantities $\tilde{S}_{I J}$ that determine the $P_{n a d}$ in models of considered type. In Section III we also derive the gauge invariant equations for variables that describe the evolution of non-adiabatic density and pressure perturbations. Some applications of the proposed formalism are discussed in Section [V] In particular, the case of two minimally coupled fluids and applications to cosmological models with coupled dark matter and quintessence dark energy is studied in detail. We conclude the paper in Section $\mathrm{V}$.

\section{BASIC EQUATIONS.}

Let us consider scalar type perturbations at a FRW background. The general line element for scalar perturbations is

$$
d s^{2}=a^{2}(\tau)\left\{-(1+2 \phi) d \tau^{2}+2 B_{, i} d \tau d x^{i}+\left[(1-2 \psi) \delta_{i j}+2 E_{, i j}\right] d x^{i} d x^{j}\right\},
$$

where four scalar $\phi, \psi, B, E$ are the first-order quantities and $\tau$ is the conformal time.

A perfect $I$-fluid with density $\rho_{I}=\bar{\rho}_{I}+\delta \rho_{I}$, pressure $P_{I}=\bar{P}_{I}+\delta P_{I}$, four-velocity $u_{I}^{\mu}=d x_{I}^{\mu} / d s$ and vanishing anisotropic stress is defined to have an energy-momentum tensor of the form

$$
T_{I \nu}^{\mu}=\left(\rho_{I}+P_{I}\right) u_{I}^{\mu} u_{I \nu}+P_{I} \delta_{\nu}^{\mu},
$$

The perturbed four-velocity can be written to the first-order terms as

$$
u_{I}^{\mu}=\frac{1}{a}\left[(1-\phi), v_{I}^{, i}\right], \quad u_{I \nu}=a\left[-(1+\phi), v_{I, i}+B_{, i}\right] .
$$

Here, we introduce the velocity potential $v_{I}$, since the fluid flow is irrotational for scalar perturbations.

Throughout this paper, we will work in the Fourier space. The first-order perturbed Einstein equations yield

$$
\begin{aligned}
3 \mathcal{H}\left(\psi^{\prime}+\mathcal{H} \phi\right)+k^{2} \psi+k^{2} \mathcal{H}\left(E^{\prime}-B\right) & =-4 \pi G a^{2} \delta \rho, \\
\psi^{\prime}+\mathcal{H} \phi & =-4 \pi G a^{2}(\bar{\rho}+\bar{P})\left(B+v_{I}\right), \\
\psi^{\prime \prime}+2 \mathcal{H} \psi^{\prime}+\mathcal{H} \phi^{\prime}+\left(2 \mathcal{H}^{\prime}+\mathcal{H}^{2}\right) \phi & =4 \pi G a^{2} \delta P, \\
\left(E^{\prime}-B\right)^{\prime}+2 \mathcal{H}\left(E^{\prime}-B\right)-\phi+\psi & =0,
\end{aligned}
$$

where $k$ is a comoving wave number, the prime denotes the differentiation with respect to the conformal time $\tau$, and $\mathcal{H}=a^{\prime} / a$.

In the general case of $N$ coupled fluids divergence of the energy-momentum tensor gives

$$
T_{I ; \nu}^{\mu \nu}=Q_{I}^{\mu}
$$

The four-vectors $Q_{A}^{\mu}$ are related by the constraint [1]

$$
\sum_{I=1}^{N} Q_{I}^{\mu}=0
$$

which results from the conservation law of the total energy-momentum tensor. For convenience, one can decompose these 4 -vectors into two parts

$$
Q_{I}^{\mu}=Q_{I} u^{\mu}+F_{I}^{\mu}, \quad Q_{I}=\bar{Q}_{I}+\delta Q_{I}, \quad u_{\mu} F_{I}^{\mu}=0 .
$$

Here $u^{\mu}$ is the overall four-velocity, $Q_{I}$ is the energy density transfer rate and $F_{I}^{\mu}$ is the momentum density transfer rate of $I$-fluid in the total matter gauge. We have $F_{I}^{\mu}=a^{-1}\left(0, f_{I}{ }^{i}\right)$ up to first-order, where $f_{I}$ is a momentum transfer potential [12].

The continuity equations for coupled perfect fluids can be obtained by the linearization of conservation equations (8). As a result, one can write the background

$$
\bar{\rho}_{I}^{\prime}=-3 \mathcal{H}\left(1+w_{I}\right) \bar{\rho}_{I}+a \bar{Q}_{I}
$$


and the perturbed equations [12]

$$
\begin{aligned}
\delta \rho_{I}^{\prime}+3 \mathcal{H}\left(\delta \rho_{I}+\delta P_{I}\right)-3\left(\bar{\rho}_{I}+\bar{P}_{I}\right) \psi^{\prime}-k^{2}\left(\bar{\rho}_{I}+\bar{P}_{I}\right)\left(v_{I}+E^{\prime}\right) & =a \bar{Q}_{I} \phi+a \delta Q_{I}, \\
{\left[\left(\bar{\rho}_{I}+\bar{P}_{I}\right)\left(v_{I}+B\right)\right]^{\prime}+4 \mathcal{H}\left(\bar{\rho}_{I}+\bar{P}_{I}\right)\left(v_{I}+B\right)+\left(\bar{\rho}_{I}+\bar{P}_{I}\right) \phi+\delta P_{I} } & =a \bar{Q}_{I}(v+B)+a f_{I} .
\end{aligned}
$$

Pressure perturbations can be expressed in terms of density and velocity perturbations as [12]

$$
\delta P_{I}=c_{s A}^{2} \delta \rho_{I}-\left(c_{s I}^{2}-c_{s I(a d)}^{2}\right) \rho_{I}^{\prime} \frac{\theta_{I}}{k^{2}} .
$$

where the velocity perturbation $\theta_{I}=-k^{2}\left(B+v_{I}\right), c_{I s(a d)}^{2}=P_{I}^{\prime} / \rho_{I}^{\prime}$ is the $I$-fluid adiabatic sound speed and $c_{s I}^{2}=$ $\delta P_{I} / \delta \rho_{I}$ is defined in the $I$-fluid rest frame. The values of $c_{I s(a d)}^{2}$ and $c_{s I}^{2}$ are equal for the barotropic fluid (i.e. fluid for which the pressure depends only on the density), but this equality may not be satisfied in a general case. For example, for a canonical scalar field one has to set $c_{s I}^{2}=1[10]$.

Using the notation

$$
q_{I} \equiv \frac{a \bar{Q}_{I}}{3 \mathcal{H}\left(\bar{\rho}_{I}+\bar{p}_{I}\right)}
$$

and the identity

$$
w_{I}^{\prime}=\left(\frac{\bar{P}_{I}}{\bar{\rho}_{I}}\right)^{\prime}=3 \mathcal{H}\left(1+w_{I}\right)\left(1-q_{I}\right)\left(w_{I}-c_{s I(a d)}^{2}\right)
$$

the equations (12), (13) can be rewritten as

$$
\begin{aligned}
\left(\Delta_{I}-3 \psi\right)^{\prime}+ & 3 \mathcal{H}\left(c_{s I}^{2}-c_{s I(a d)}^{2}+q_{I}\left(c_{s I(a d)}^{2}+1\right)\right) \Delta_{I} \\
& +9 \mathcal{H}^{2}\left(c_{s I}^{2}-c_{s I(a d)}^{2}\right)\left(1-q_{I}\right) \frac{\theta_{I}}{k^{2}}+\theta_{I}-k^{2}\left(E^{\prime}-B\right)=3 \mathcal{H} q_{I}\left(\phi+\frac{\delta Q_{I}}{\bar{Q}_{I}}\right), \\
\frac{\theta_{I}^{\prime}}{k^{2}}+\mathcal{H} \frac{\theta_{I}}{k^{2}}= & c_{s I}^{2}\left(\Delta_{I}+3 \mathcal{H}\left(1-q_{I}\right) \frac{\theta_{I}}{k^{2}}\right)+\phi+3 \mathcal{H} q_{I}\left(\frac{\theta}{k^{2}}-\frac{\theta_{I}}{k^{2}}\right)-\frac{a}{\rho_{I}\left(1+w_{I}\right)} f_{I},
\end{aligned}
$$

where $\Delta_{I}=\delta \rho_{I} /\left(\bar{\rho}_{I}+\bar{P}_{I}\right)$.

\section{NON-ADIABATIC PERTURBATIONS.}

The comoving curvature perturbation $\mathcal{R}=\psi-\mathcal{H}(v+B)$ at spatially flat background can be represented in terms of the longitudinal gauge-invariant quantities [22]

$$
\Phi \equiv \phi+\mathcal{H}\left(B-E^{\prime}\right)+\left(B-E^{\prime}\right)^{\prime}, \quad \Psi \equiv \psi-\mathcal{H}\left(B-E^{\prime}\right)
$$

as

$$
\mathcal{R}=\Psi+\frac{2}{3} \frac{\Psi^{\prime}+\mathcal{H} \Phi}{\mathcal{H}(1+w)}
$$

The time derivative of equation (20) gives the well known equation

$$
\mathcal{R}^{\prime}=\frac{2}{3} \frac{1}{\mathcal{H}(1+w)}\left\{\frac{\kappa^{2}}{2} a^{2} P_{n a d}-k^{2} c_{s(a d)}^{2} \Psi\right\},
$$

where the non-adiabatic part of the pressure perturbation is defined by

$$
P_{n a d}=\delta P-c_{s(a d)}^{2} \delta \rho .
$$


Substituting the expression $c_{s(a d)}^{2}=\sum_{I} \frac{\bar{\rho}_{I}^{\prime}}{\bar{\rho}^{\prime}} c_{s I(a d)}^{2}$ for the overall adiabatic sound speed, at the arbitrary number of coupled perfect fluids, one can obtain

$$
\begin{aligned}
P_{n a d}= & \sum_{I} \frac{\bar{\rho}_{I}^{\prime}}{\bar{\rho}^{\prime}}\left(c_{s I}^{2}-c_{s I(a d)}^{2}\right) \epsilon_{m}+\sum_{I}\left(\bar{\rho}_{I}+\bar{P}_{I}\right) c_{s I}^{2} q_{I} \Delta \\
& +\sum_{I, J} \frac{\left(\bar{\rho}_{I}+\bar{P}_{I}\right)\left(\bar{\rho}_{J}+\bar{P}_{J}\right)}{\bar{\rho}+\bar{P}}\left(c_{s I}^{2} \Delta_{I J}+3 \mathcal{H}\left(c_{s I}^{2}-c_{s I(a d)}^{2}\right)\left(1-q_{I}\right) \frac{\theta_{I J}}{k^{2}}\right)
\end{aligned}
$$

where $\Delta=\delta \rho /(\bar{\rho}+\bar{P}), \Delta_{I J}=\Delta_{I}-\Delta_{J}$, and the gauge-invariant quantity

$$
\epsilon_{m} \equiv \delta \rho+3 \mathcal{H}(\bar{\rho}+\bar{P}) \frac{\theta}{k^{2}}
$$

is a comoving density perturbation 23].

The equation (23) contains quantities $\Delta_{I J}$ that are not gauge invariant at non-minimal coupling. Hence, following Refs. [11], [20], 24], it is convenient to use the gauge invariant variables

$$
\hat{\Delta}_{I J}=\frac{\delta \rho_{I}}{\left(1-q_{I}\right)\left(\bar{\rho}_{I}+\bar{P}_{I}\right)}-\frac{\delta \rho_{J}}{\left(1-q_{J}\right)\left(\bar{\rho}_{J}+\bar{P}_{J}\right)}
$$

or

$$
\tilde{\Delta}_{I J}=\left(1-q_{I}\right)\left(1-q_{J}\right) \hat{\Delta}_{I J}
$$

instead. The latter quantities are well defined even at $q_{I}=1$.

In these new variables, the expression (23) can be rewritten as

$$
P_{n a d}=\sum_{I} \frac{\bar{\rho}_{I}^{\prime}}{\bar{\rho}^{\prime}}\left(c_{s I}^{2}-c_{s I(a d)}^{2}\right) \epsilon_{m}+\frac{1}{2} \sum_{I, J} \frac{\left(\bar{\rho}_{I}+\bar{P}_{I}\right)\left(\bar{\rho}_{J}+\bar{P}_{J}\right)}{\bar{\rho}+\bar{P}} \tilde{S}_{I J},
$$

where

$$
\tilde{S}_{I J}=\left(c_{s I}^{2}-c_{s J}^{2}\right) \tilde{\Delta}_{I J}+3 \mathcal{H}\left(\left(c_{s I}^{2}-c_{s I(a d)}^{2}\right)\left(1-q_{I}\right)-\left(c_{s J}^{2}-c_{s J(a d)}^{2}\right)\left(1-q_{J}\right)\right) \frac{\theta_{I J}}{k^{2}} .
$$

The perturbed Einstein equations (4) and (5) lead to the relation

$$
\frac{k^{2}}{a^{2}} \Psi=-4 \pi G \epsilon_{m}
$$

and in the long-wavelength limit $k \rightarrow 0$ we obtain $\epsilon_{m} \rightarrow 0$. Hence, the terms with $\epsilon_{m}$ in the equation (27) can be neglected on a large scale.

The symmetrical quantities $\tilde{S}_{I J}$ are closely related to the entropy perturbations and adiabatic condition. Using the definition of the scalar field energy-momentum tensor, the adiabaticity condition for systems of canonical scalar fields $\varphi_{I}[22]$

$$
\frac{\delta \varphi_{I}}{\varphi_{I}^{\prime}}-\frac{\delta \varphi_{J}}{\varphi_{J}^{\prime}}=0
$$

can be rewritten as

$$
\frac{\theta_{I J}}{k^{2}}=0
$$

where $I, J=1, \ldots, N$.

The adiabatic mode in multi-fluid models with barotropic coupled fluids is determined by the conditions [24]

$$
\hat{\Delta}_{I J}=0 .
$$

In both cases, quantities $\tilde{S}_{I J}$ vanish. The case of systems with barotropic fluids and scalar fields is more complicated, since the leading contributions to $\tilde{S}_{I J}$ depend on the gauge choice. In cosmological models with minimally coupled 
quintessence dark energy, one can put $\tilde{S}_{I J}=0$ for the growing adiabatic mode in the longitudinal gauge, but some of quantities $\tilde{S}_{I J}$ are different from zero at leading order in $k \tau$ at "generalized initial adiabatic conditions" of [21] in the synchronous gauge. The "generalized initial adiabatic conditions" provide only that the inequality $P_{n a d} \ll \delta p$ holds. In any case, to find $P_{n a d}$ and the comoving curvature perturbation $\mathcal{R}$, it is necessary to know the values of $\tilde{S}_{I J}$.

The equation (27) shows that the non-adiabatic pressure perturbation depend on $\hat{\Delta}_{I J}$ and $\theta_{I J}$ through their linear combinations $\tilde{S}_{I J}$. This fact allows to simplify the analysis of the evolution of the non-adiabatic pressure perturbation that is important for the study of possibility of the non-adiabatic early instabilities in the models with coupled fluids.

The quantities $\tilde{S}_{I J}$ are completely determined by the values of $\hat{\Delta}_{I J}$ and $\theta_{I J}$. Following Refs. [24],[20],[11], we introduce the notations

$$
\hat{\epsilon}_{I}=\frac{\delta Q_{I}}{\bar{Q}_{I}}+\frac{\bar{Q}_{I}^{\prime}}{3 \mathcal{H} \bar{Q}_{I}} \Delta, \quad f_{I J}=\frac{a f_{I}}{\rho_{I}\left(1+w_{I}\right)}-\frac{a f_{J}}{\rho_{J}\left(1+w_{J}\right)}, \quad \hat{E}_{I J}=\frac{q_{I} \hat{\epsilon}_{I}}{1-q_{I}}-\frac{q_{J} \hat{\epsilon}_{J}}{1-q_{J}} .
$$

Then for the system of $N$ coupled fluids (taking into account $\rho=\sum_{I} \rho_{I}$ ) the equations (17), (18) yield

$$
\begin{aligned}
\hat{\Delta}_{I J}^{\prime}- & \frac{1}{2}\left(\frac{q_{I}^{\prime}}{1-q_{I}}-3 \mathcal{H}\left(c_{s I}^{2}-c_{s I(a d)}^{2}\right)-3 \mathcal{H} q_{I}\left(c_{s I(a d)}^{2}+1\right)\right) \sum_{K} \frac{\bar{\rho}_{K}^{\prime}}{\bar{\rho}^{\prime}}\left(\hat{\Delta}_{I K}+\hat{\Delta}_{J K}\right) \\
& +\frac{1}{2}\left(\frac{q_{J}^{\prime}}{1-q_{J}}-3 \mathcal{H}\left(c_{s J}^{2}-c_{s J(a d)}^{2}\right)-3 \mathcal{H} q_{J}\left(c_{s J(a d)}^{2}+1\right)\right) \sum_{K} \frac{\bar{\rho}_{K}^{\prime}}{\bar{\rho}^{\prime}}\left(\hat{\Delta}_{I K}+\hat{\Delta}_{J K}\right) \\
& +\frac{3}{2} \mathcal{H}\left(c_{s I}^{2}-c_{s I(a d)}^{2}+c_{s J}^{2}-c_{s J(a d)}^{2}+q_{I}\left(c_{s I(a d)}^{2}+1\right)+q_{J}\left(c_{s J(a d)}^{2}+1\right)\right) \hat{\Delta}_{I J} \\
& -\frac{1}{2}\left(\frac{q_{I}^{\prime}}{1-q_{I}}+\frac{q_{J}^{\prime}}{1-q_{J}}\right) \hat{\Delta}_{I J}+\frac{9}{2} \mathcal{H}^{2}\left(c_{s I}^{2}-c_{s I(a d)}^{2}+c_{s J(a d)}^{2}-c_{s J}^{2}\right) \sum_{K} \frac{\bar{\rho}_{K}+\bar{P}_{K}}{\bar{\rho}+\bar{P}}\left(\frac{\theta_{I K}}{k^{2}}+\frac{\theta_{J K}}{k^{2}}\right) \\
& =3 \mathcal{H} \hat{E}_{I J}-\left(\frac{1}{1-q_{I}}-\frac{1}{1-q_{J}}\right) \theta_{u c}-3 \mathcal{H}\left(c_{s I}^{2}-c_{s I(a d)}^{2}+c_{s J(a d)}^{2}-c_{s J}^{2}\right) \frac{\epsilon_{m}}{\bar{\rho}+\bar{P}}, \\
& \frac{3}{2}\left(c_{s I}^{2}-c_{s J}^{2}-q_{I}\left(1+c_{s I}^{2}\right)+q_{J}\left(1+c_{s J}^{2}\right)\right) \sum_{K} \frac{\bar{\rho}_{K}+\bar{P}_{K}}{\theta_{I J}+\bar{P}^{\prime}}\left(\frac{\theta_{I K}}{k^{2}}+\frac{\theta_{J K}}{k^{2}}\right) \\
& +\mathcal{H}\left(1-\frac{3}{2} c_{s I}^{2}-\frac{3}{2} c_{s J}^{2}+\frac{3}{2} q_{I}\left(1+c_{s I}^{2}\right)+\frac{3}{2} q_{J}\left(1+c_{s J}^{2}\right)\right) \frac{\theta_{I J}}{k^{2}} \\
& -\frac{1}{2}\left(c_{s I}^{2}\left(1-q_{I}\right)-c_{s B}^{2}\left(1-q_{J}\right)\right) \sum_{K} \frac{\bar{\rho}_{K}^{\prime}}{\bar{\rho}^{\prime}}\left(\hat{\Delta}_{I K}+\hat{\Delta}_{J K}\right) \\
& -\frac{1}{2}\left(c_{s I}^{2}\left(1-q_{I}\right)+c_{s J}^{2}\left(1-q_{J}\right)\right) \hat{\Delta}_{I J}=\left(\left(1-q_{I}\right) c_{s I}^{2}-\left(1-q_{J}\right) c_{s J}^{2}\right) \frac{\epsilon_{m}}{\bar{\rho}+\bar{P}}-f_{I J},
\end{aligned}
$$

where $I, J, K=1, \ldots, N$ and

$$
\theta_{u c} \equiv \theta+k^{2} \frac{\psi}{\mathcal{H}}
$$

is the velocity perturbation on uniform curvature hypersurfaces.

These equations agree with the equations of Ref. [24] on a spatially flat background at a vanishing anisotropic stress ${ }^{1}$. On a large scale, after substituting the explicit expressions for $\hat{E}_{A B}$ and $f_{A B}$, the equations (34), (35) form a closed system of equations.

The system of equations (34), (35) contains $N(N-1)$ independent equations for the antisymmetric quantities $\hat{\Delta}_{I J}$ and $\theta_{I J}$. Meanwhile, for the analysis of the evolution of the non-adiabatic pressure perturbation, it is suffices to know only the values of $N(N-1) / 2$ symmetric quantities $\tilde{S}_{I J}$. In many cases of practical importance, on a large scale, it is not need to solve the complete system of equations (34) and (35), and one can derive and solve the equations for $\tilde{S}_{I J}$. In what follows, we consider some applications of the new variables.

\footnotetext{
1 There is a typo in the common sign of the second line of Eq. (2.196) of Ref. [24].
} 


\section{SEVERAL MODELS.}

\section{A. Two non-coupled fluids.}

Models with non-interacting fractions are often used in modern cosmology, especially in inflationary models. They are of interest as toy-models when considering the radiation dominated universe. Primarily, we are interested in studying the influence of non-adiabatic sound speed on rate of decay of non-adiabatic perturbations in cosmological models with quintessence. We would also like to clarify the general arguments [25] that the non-adiabatic perturbations evolve independently of the adiabatic ones within the effective fluid formalism applied in the paper.

\section{Non coupled barotropic fluid and scalar field.}

Consider a simple model with two minimally coupled perfect fluids, indicated by subscripts $A$ and $B$, where the $B$-component is a barotropic fluid. In this case, the equations (334), (35) are reduced to

$$
\begin{aligned}
\tilde{\Delta}_{A B}^{\prime}+3 \mathcal{H}\left(c_{s A}^{2}-c_{s A(a d)}^{2}\right) \frac{\bar{\rho}_{B}^{\prime}}{\bar{\rho}^{\prime}}\left(\tilde{\Delta}_{A B}+3 \mathcal{H} \frac{\theta_{A B}}{k^{2}}\right)+\theta_{A B}=-3 \mathcal{H}\left(c_{s A}^{2}-c_{s A(a d)}^{2}\right) \frac{\epsilon_{m}}{\bar{\rho}+\bar{P}}, \\
\frac{\theta_{A B}^{\prime}}{k^{2}}+\mathcal{H} \frac{\theta_{A B}}{k^{2}}-\left(c_{s A}^{2} \frac{\bar{\rho}_{B}^{\prime}}{\bar{\rho}^{\prime}}+c_{s B}^{2} \frac{\bar{\rho}_{A}^{\prime}}{\bar{\rho}^{\prime}}\right)\left(\tilde{\Delta}_{A B}+3 \mathcal{H} \frac{\theta_{A B}}{k^{2}}\right)=\left(c_{s A}^{2}-c_{s B}^{2}\right) \frac{\epsilon_{m}}{\bar{\rho}+\bar{P}} .
\end{aligned}
$$

The definition (28) gives

$$
\tilde{S}_{A B}=\left(c_{s A}^{2}-c_{s B}^{2}\right) \tilde{\Delta}_{A B}+3 \mathcal{H}\left(c_{s A}^{2}-c_{s A(a d)}^{2}\right) \frac{\theta_{A B}}{k^{2}} .
$$

Here we restrict ourselves only to the case of constant $c_{s A}^{2}, c_{s B}^{2}, w_{A}, w_{B}$. At constant parameters $w_{A}, w_{B}$ the equation (16) implies the relations

$$
c_{s A(a d)}^{2}=w_{A}, \quad c_{s B(a d)}^{2}=w_{B} .
$$

The equations (37), (38) can be combined to form the second order equation

$$
\begin{aligned}
\tilde{S}_{A B}^{\prime \prime}+ & \mathcal{H}\left[1+3\left(c_{s A}^{2} \frac{\bar{\rho}_{B}^{\prime}}{\bar{\rho}^{\prime}}+c_{s B}^{2} \frac{\bar{\rho}_{A}^{\prime}}{\bar{\rho}^{\prime}}\right) \frac{c_{s B}^{2}-c_{s A(a d)}^{2}}{c_{s A}^{2}-c_{s B}^{2}}-3 c_{s B}^{2} \frac{c_{s A}^{2}-c_{s A(a d)}^{2}}{c_{s A}^{2}-c_{s B}^{2}}-\frac{\xi^{\prime}}{\mathcal{H} \xi}\right] \tilde{S}_{A B}^{\prime} \\
& +3 \mathcal{H}^{2}\left[\left(c_{s A}^{2} \frac{\bar{\rho}_{B}^{\prime}}{\bar{\rho}^{\prime}}+c_{s B}^{2} \frac{\bar{\rho}_{A}^{\prime}}{\bar{\rho}^{\prime}}\right)\left(1-\frac{\mathcal{H}^{\prime}}{\mathcal{H}^{2}}\right)-\left(1+\frac{\mathcal{H}^{\prime}}{\mathcal{H}^{2}}-\frac{\xi^{\prime}}{\mathcal{H} \xi}\right) c_{s B}^{2}\right] \frac{c_{s A}^{2}-c_{s A(a d)}^{2}}{c_{s A}^{2}-c_{s B}^{2}} \tilde{S}_{A B} \\
& +\left(c_{s A}^{2} \frac{\bar{\rho}_{B}^{\prime}}{\bar{\rho}^{\prime}}+c_{s B}^{2} \frac{\bar{\rho}_{A}^{\prime}}{\bar{\rho}^{\prime}}\right) k^{2} \tilde{S}_{A B}=\xi\left(c_{s A}^{2}-c_{s B}^{2}\right) \frac{\epsilon_{m}}{\bar{\rho}+\bar{P}}
\end{aligned}
$$

where

$$
\xi=-\left[\left(c_{s A}^{2}-c_{s B}^{2}\right) k^{2}+3 \mathcal{H}^{2}\left(1-\frac{\mathcal{H}^{\prime}}{\mathcal{H}^{2}}+3 c_{s B}^{2} \frac{c_{s B}^{2}-c_{s A(a d)}^{2}}{c_{s A}^{2}-c_{s B}^{2}}\right)\left(c_{s A}^{2}-c_{s A(a d)}^{2}\right)\right] .
$$

For two barotropic fluids with the indices $A$ and $B$, the equation (41) is reduced to

$$
\tilde{S}_{A B}^{\prime \prime}+\mathcal{H}\left[1-3\left(c_{s A}^{2} \frac{\bar{\rho}_{B}^{\prime}}{\bar{\rho}^{\prime}}+c_{s B}^{2} \frac{\bar{\rho}_{A}^{\prime}}{\bar{\rho}^{\prime}}\right)\right] \tilde{S}_{A B}^{\prime}+k^{2}\left(c_{s A}^{2} \frac{\bar{\rho}_{B}^{\prime}}{\bar{\rho}^{\prime}}+c_{s B}^{2} \frac{\bar{\rho}_{A}^{\prime}}{\bar{\rho}^{\prime}}\right) \tilde{S}_{A B}=-k^{2}\left(c_{s A}^{2}-c_{s B}^{2}\right)^{2} \frac{\epsilon_{m}}{\bar{\rho}+\bar{P}} .
$$

The additional multiplier $k^{2}$ on the right hand side of (43) ensures that in the large scale limit $k \rightarrow 0$, the source term is negligibly small, and the adiabatic perturbations do not affect the entropy evolution.

When the $A$-component is a canonical scalar field, the implicit form of the equation (41) looks rather complicated. We write it only for the case of radiation and subdominant scalar field. Then, on a large scale, we obtain

$$
\tilde{S}_{A B}^{\prime \prime}+3 \mathcal{H}\left(1-c_{s A(a d)}^{2}\right) \tilde{S}_{A B}^{\prime}+6 \mathcal{H}^{2}\left(1-c_{s A(a d)}^{2}\right) \tilde{S}_{A B}=-\mathcal{H}^{2}\left(5-3 c_{s A(a d)}^{2}\right)\left(1-c_{s A(a d)}^{2}\right) \frac{\epsilon_{m}}{\bar{\rho}+\bar{P}} .
$$


Although this equation is gauge invariant, according to the definition of $\tilde{S}_{A B}$, one can drop the source only if $\max \left\{\left|\Delta_{A}\right|,\left|\Delta_{B}\right|, \mathcal{H}\left|\theta_{A} / k^{2}\right|, \mathcal{H}\left|\theta_{B} / k^{2}\right|\right\} \gg \frac{\epsilon_{m}}{\rho+p}$. This condition is not satisfied, in general, in the synchronous gauge.

Indeed, for the "generalized adiabatic initial conditions" of Ref. [21]

$$
\begin{aligned}
\Delta_{A} & =-\frac{C}{2} \frac{4-3 c_{s A}^{2}}{4-6 w_{A}+3 c_{s A}^{2}}(k \tau)^{2} \\
\theta_{A} & =-\frac{C}{2} \frac{c_{s A}^{2}}{4-6 w_{A}+3 c_{s A}^{2}}(k \tau)^{3} k
\end{aligned}
$$

we get

$$
\tilde{S}_{A B}=\left(1-w_{A}\right) \frac{10-6 w_{A}}{3\left(7-6 w_{A}\right)} \frac{C}{2}(k \tau)^{2}, \quad \frac{\epsilon_{m}}{\bar{\rho}+\bar{P}}=-\frac{4}{3} \frac{C}{2}(k \tau)^{2}
$$

where $C$ is a constant. A direct verification shows that (44) holds for the "generalized adiabatic initial conditions" only if we do not neglect the source in this equation. Thus, in the synchronous gauge, the adiabatic perturbations give rise to non-adiabatic perturbations even on a large scale.

It is interesting to compare equation (43) and (44) at the radiation dominated stage in case the quantities $\tilde{S}_{A B}$ are large enough for the sources to be neglected. The equation (43) yields on a large scale

$$
\tilde{S}_{A B}^{\prime \prime}+\mathcal{H}\left(1-3 w_{A}\right) \tilde{S}_{A B}^{\prime}=0 \quad \text { (fluid - fluid) }
$$

The equation (44) takes the form

$$
\tilde{S}_{A B}^{\prime \prime}+\mathcal{H}\left(3-3 w_{A}\right) \tilde{S}_{A B}^{\prime}+6 \mathcal{H}^{2}\left(1-w_{A}\right) \tilde{S}_{A B}=0 \quad \text { (scalar field - fluid). }
$$

It follows that, if the $A$-fluid is the minimally coupled scalar field, the damping force is stronger. In addition, when $w_{A}<1$, the coefficient at $\tilde{S}_{A B}$ is positive. Hence, at negative $w_{A}$, the quantity $\tilde{S}_{A B}$ rapidly approaches the asymptotic solution. For this reason, in numerical codes like, CMBFAST and CAMB, the initial dark energy perturbations are set by default to zero.

\section{Two scalar fields.}

The case of two non-interacting scalar fields, and the more general case of interacting fields are studied carefully by different methods. Here, we look at the second order equation for $\widetilde{S}_{A B}$ only. From the original equations (34) and (35), it is easy to write an equation for $\theta_{A B}$. It has the form

$$
\begin{aligned}
\frac{\theta_{A B}^{\prime \prime}}{k^{2}}+ & \mathcal{H}\left(1-3\left(c_{s A(a d)}^{2} \frac{\bar{\rho}_{B}^{\prime}}{\bar{\rho}^{\prime}}+c_{s B(a d)}^{2} \frac{\bar{\rho}_{A}^{\prime}}{\bar{\rho}^{\prime}}\right)\right) \frac{\theta_{A B}^{\prime}}{k^{2}}+\theta_{A B} \\
& +\mathcal{H}^{2}\left(3-2 \frac{\mathcal{H}^{\prime}}{\mathcal{H}^{2}}-3\left(c_{s A(a d)}^{2} \frac{\bar{\rho}_{B}^{\prime}}{\bar{\rho}^{\prime}}+c_{s B(a d)}^{2} \frac{\bar{\rho}_{A}^{\prime}}{\bar{\rho}^{\prime}}\right)\right) \frac{\theta_{A B}}{k^{2}}=3 \mathcal{H}\left(c_{s A(a d)}^{2}-c_{s B(a d)}^{2}\right) \frac{\epsilon_{m}}{\bar{\rho}+\bar{P}}
\end{aligned}
$$

At constant adiabatic sound speeds, this equation can be rewritten in terms of $\tilde{S}_{A B}$ as

$$
\begin{aligned}
\tilde{S}_{A B}^{\prime \prime}+ & \mathcal{H}\left(1-2 \frac{\mathcal{H}^{\prime}}{\mathcal{H}}-3\left(c_{s A(a d)}^{2} \frac{\bar{\rho}_{B}^{\prime}}{\bar{\rho}^{\prime}}+c_{s B(a d)}^{2} \frac{\bar{\rho}_{A}^{\prime}}{\bar{\rho}^{\prime}}\right)\right) S^{\prime}+k^{2} \tilde{S}_{A B} \\
& +\mathcal{H}^{2}\left(2 \frac{\mathcal{H}^{\prime 2}}{\mathcal{H}^{4}}-\frac{\mathcal{H}^{\prime \prime}}{\mathcal{H}^{3}}+3\left(1-c_{s A(a d)}^{2} \frac{\bar{\rho}_{B}^{\prime}}{\bar{\rho}^{\prime}}-c_{s B(a d)}^{2} \frac{\bar{\rho}_{A}^{\prime}}{\bar{\rho}^{\prime}}\right)\left(1-\frac{\mathcal{H}^{\prime}}{\mathcal{H}^{2}}\right)\right) \tilde{S}_{A B} \\
& =-9 \mathcal{H}^{2}\left(c_{s A(a d)}^{2}-c_{s B(a d)}^{2}\right)^{2} \frac{\epsilon_{m}}{\bar{\rho}+\bar{P}} .
\end{aligned}
$$

In general, one has to take into account the source term even on a large scale, but usually the scalar fields are considered in the longitudinal $(E=B=0)$ gauge. The well known long-wavelength adiabatic solution in this gauge [16, 17] can be rewritten as

$$
\phi=\psi=C\left(1-\frac{\mathcal{H}}{a^{2}} \int_{\tau_{1}}^{\tau} a^{2} d \tau\right)
$$




$$
\begin{aligned}
\Delta_{A} & =\Delta_{B}=-3 C \frac{\mathcal{H}}{a^{2}} \int_{\tau_{1}}^{\tau} a^{2} d \tau, \\
\frac{\theta_{A}}{k^{2}} & =\frac{\theta_{B}}{k^{2}}=\frac{C}{a^{2}} \int_{\tau_{1}}^{\tau} a^{2} d \tau,
\end{aligned}
$$

where $C$ and $\tau_{1}$ are constants. By the definition (24), the value of $\epsilon_{m}$ vanishes now in the leading order, and the right hand side of the equation (51) can be neglected.

\section{B. Special case of three fluids.}

Consider the Universe filled with quintessence dark energy $(A)$, dark matter $(B)$ and radiation $(C)$, and assume that the dark energy and the dark matter couple. Since the variables $\hat{\Delta}_{I J}, \theta_{I J}$ are antisymmetric and constrained by

$$
\hat{\Delta}_{A B}+\hat{\Delta}_{B C}+\hat{\Delta}_{C A}=0, \quad \theta_{A B}+\theta_{B C}+\theta_{C A}=0
$$

there are only four independent variables among them.

Using the relations (55) and the equalities $c_{s B}^{2}=c_{s B(a d)}^{2}, c_{s C}^{2}=c_{s C(a d)}^{2}$, which are valid for barotropic fluids, the equations (34) and (35) can be reduced to set

$$
\begin{aligned}
& \hat{\Delta}_{A C}^{\prime}-\left(\frac{q_{A}^{\prime}}{1-q_{A}}-3 \mathcal{H}\left(c_{s A}^{2}-c_{s A(a d)}^{2}\right)-3 \mathcal{H} q_{A}\left(c_{s A(a d)}^{2}+1\right)\right)\left(\frac{\bar{\rho}_{B}^{\prime}+\bar{\rho}_{C}^{\prime}}{\bar{\rho}^{\prime}} \hat{\Delta}_{A C}-\frac{\bar{\rho}_{B}^{\prime}}{\bar{\rho}^{\prime}} \hat{\Delta}_{B C}\right) \\
& +9 \mathcal{H}^{2}\left(c_{s A}^{2}-c_{s A(a d)}^{2}\right)\left(\frac{\bar{\rho}_{B}+\bar{P}_{B}+\bar{\rho}_{C}+\bar{P}_{C}}{\bar{\rho}+\bar{P}} \frac{\theta_{A C}}{k^{2}}-\frac{\bar{\rho}_{B}+\bar{P}_{B}}{\bar{\rho}+\bar{P}} \frac{\theta_{B C}}{k^{2}}\right) \\
& +\left(\frac{\bar{\rho}_{B}+\bar{P}_{B}+\bar{\rho}_{C}+\bar{P}_{C}}{\bar{\rho}+\bar{P}} \frac{1}{1-q_{A}}+\frac{\bar{\rho}_{A}+\bar{P}_{A}}{\bar{\rho}+\bar{P}}\right) \theta_{A C}-\frac{q_{A}}{1-q_{A}} \frac{\bar{\rho}_{B}+\bar{P}_{B}}{\bar{\rho}+\bar{P}} \theta_{B C} \\
& =3 \mathcal{H} \hat{E}_{A C}-\frac{q_{A}}{1-q_{A}} \theta_{u c}-3 \mathcal{H}\left(c_{s A}^{2}-c_{s A(a d)}^{2}\right) \frac{\epsilon_{m}}{\bar{\rho}+\bar{P}}, \\
& \frac{\theta_{A C}^{\prime}}{k^{2}}+\mathcal{H}\left(1-3 c_{s C}^{2}\right) \frac{\theta_{A C}}{k^{2}}-3 \mathcal{H}\left(c_{s A}^{2}-c_{s C}^{2}-q_{A}\left(1+c_{s A}^{2}\right)\right)\left(\frac{\bar{\rho}_{B}+\bar{P}_{B}+\bar{\rho}_{C}+\bar{P}_{C}}{\bar{\rho}+\bar{P}} \frac{\theta_{A C}}{k^{2}}-\frac{\bar{\rho}_{B}+\bar{P}_{B}}{\bar{\rho}+\theta_{B C}} \frac{\theta_{P}}{k^{2}}\right) \\
& -\left(\left(1-q_{A}\right) c_{s A}^{2}-c_{s C}^{2}\right)\left(\frac{\bar{\rho}_{B}^{\prime}+\bar{\rho}_{C}^{\prime}}{\bar{\rho}^{\prime}} \hat{\Delta}_{A C}-\frac{\bar{\rho}_{B}^{\prime}}{\bar{\rho}^{\prime}} \hat{\Delta}_{B C}\right)-c_{s C}^{2} \hat{\Delta}_{A C}=\left(\left(1-q_{A}\right) c_{s A}^{2}-c_{s C}^{2}\right) \frac{\epsilon_{m}}{\bar{\rho}+\bar{P}}-f_{A C}, \\
& \hat{\Delta}_{B C}^{\prime}-\left(\frac{q_{B}^{\prime}}{1-q_{B}}-3 \mathcal{H} q_{B}\left(c_{s B(a d)}^{2}+1\right)\right)\left(\frac{\bar{\rho}_{A}^{\prime}+\bar{\rho}_{C}^{\prime}}{\bar{\rho}^{\prime}} \hat{\Delta}_{B C}-\frac{\bar{\rho}_{A}^{\prime}}{\bar{\rho}^{\prime}} \hat{\Delta}_{A C}\right) \\
& +\left(\frac{\bar{\rho}_{B}+\bar{P}_{B}}{\rho+p}+\frac{\bar{\rho}_{A}+\bar{P}_{A}+\bar{\rho}_{C}+\bar{P}_{C}}{\bar{\rho}+\bar{P}} \frac{1}{1-q_{B}}\right) \theta_{B C}-\frac{\bar{\rho}_{A}+\bar{P}_{A}}{\bar{\rho}+\bar{P}} \frac{q_{B}}{1-q_{B}} \theta_{A C}=3 \mathcal{H} \hat{E}_{B C}-\frac{q_{B}}{1-q_{B}} \theta_{u c} \\
& \frac{\theta_{B C}^{\prime}}{k^{2}}+\mathcal{H}\left(1-3 c_{s C}^{2}\right) \frac{\theta_{B C}}{k^{2}}-3 \mathcal{H}\left(c_{s B}^{2}-c_{s C}^{2}-q_{B}\left(1+c_{s B}^{2}\right)\right)\left(\frac{\bar{\rho}_{A}+\bar{P}_{A}+\bar{\rho}_{C}+\bar{P}_{C}}{\bar{\rho}+\bar{P}} \frac{\theta_{B C}}{k^{2}}-\frac{\bar{\rho}_{A}+\bar{P}_{A}}{\bar{\rho}+\bar{P}} \frac{\theta_{A C}}{k^{2}}\right) \\
& -\left(\left(1-q_{B}\right) c_{s B}^{2}-c_{s C}^{2}\right)\left(\frac{\bar{\rho}_{A}^{\prime}+\bar{\rho}_{C}^{\prime}}{\bar{\rho}^{\prime}} \hat{\Delta}_{B C}-\frac{\bar{\rho}_{A}^{\prime}}{\bar{\rho}^{\prime}} \hat{\Delta}_{A C}\right)-c_{s C}^{2} \hat{\Delta}_{B C}=\left(\left(1-q_{B}\right) c_{s B}^{2}-c_{s C}^{2}\right) \frac{\epsilon_{m}}{\bar{\rho}+\bar{P}}-f_{B C}
\end{aligned}
$$

At the radiation dominated stage the dark energy and dark matter are subdominant, $\frac{\rho_{A}}{\rho} \ll 1$, $\frac{\rho_{B}}{\rho} \ll 1$, which allows to simplify the equations. Furthermore, we assume that we can neglect terms with $\frac{\epsilon_{m}}{\rho+p}$ in the long-wavelength limit. As a result, on a large scale, we obtain the approximate equations

$$
\begin{aligned}
& \tilde{\Delta}_{A C}^{\prime}+3 \mathcal{H}\left(c_{s A}^{2}-c_{s A(a d)}^{2}+q_{A}\left(c_{s A(a d)}^{2}+1\right)\right) \tilde{\Delta}_{A C}+9 \mathcal{H}^{2}\left(c_{s A}^{2}-c_{s A(a d)}^{2}\right)\left(1-q_{A}\right) \frac{\theta_{A C}}{k^{2}}=3 \mathcal{H}\left(1-q_{A}\right) \hat{E}_{A C}, \\
& \frac{\theta_{A C}^{\prime}}{k^{2}}+\mathcal{H}\left(1-3 c_{s A}^{2}+3 q_{A}\left(1+c_{s A}^{2}\right)\right) \frac{\theta_{A C}}{k^{2}}-c_{s A}^{2} \tilde{\Delta}_{A C}=-f_{A C}, \\
& \tilde{\Delta}_{B C}^{\prime}+3 \mathcal{H} q_{B}\left(c_{s B(a d)}^{2}+1\right) \tilde{\Delta}_{B C}+\theta_{B C}+q_{B} \theta_{u c}=3 \mathcal{H}\left(1-q_{B}\right) \hat{E}_{B C}, \\
& \frac{\theta_{B C}^{\prime}}{k^{2}}+\mathcal{H}\left(1-3 c_{s B}^{2}+3 q_{B}\left(1+c_{s B}^{2}\right)\right) \frac{\theta_{B C}}{k^{2}}-c_{s B}^{2} \tilde{\Delta}_{B C}=-f_{B C} .
\end{aligned}
$$

In the following we explicitly use the values $c_{s A}^{2}=1, c_{s B}^{2}=0, c_{s C}^{2}=1 / 3$. 


\section{Example 1.}

As a simple model, consider the coupling [26]

$$
Q^{\mu}=\gamma \rho_{A} \rho_{B}\left(u_{B}^{\mu}-u_{A}^{\mu}\right)
$$

where $\gamma$ is a constant.

For such interaction, all the quantities $\bar{Q}_{A}$ are zero and the evolution of background variables is the same as at minimally coupled fractions. Now $q_{A}=q_{B}=\epsilon_{A}=\epsilon_{B}=0$ and

$$
f_{A C} \approx \frac{a \gamma \bar{\rho}_{B}}{1+w_{A}} \frac{\theta_{A B}}{k^{2}}, \quad f_{B C} \approx-a \gamma \bar{\rho}_{A} \frac{\theta_{A B}}{k^{2}} .
$$

The equations (62) and (63) give

$$
\begin{aligned}
\hat{\Delta}_{B C}^{\prime}+\theta_{B C} & =0, \\
\frac{\theta_{B C}^{\prime}}{k^{2}}+\mathcal{H} \frac{\theta_{B C}}{k^{2}} & =a \gamma \bar{\rho}_{A} \frac{\theta_{A B}}{k^{2}} .
\end{aligned}
$$

We consider here only the case of $w_{A}<-1 / 3$. Since for minimally coupled fractions $\bar{\rho}_{A} \propto a^{-3\left(1+w_{A}\right)}$ and $\left|\frac{a \gamma \bar{\rho}_{A}}{\mathcal{H}}\right| \ll 1$ at early times, we find that one can consistently assume that $\tilde{\Delta}_{B C}=\theta_{B C}=0$ if $\theta_{A B}$ are not very large. Then the first two equations, (60) and (61), are simplified to

$$
\begin{aligned}
\tilde{\Delta}_{A C}^{\prime}+3 \mathcal{H}\left(1-w_{A}\right) \tilde{\Delta}_{A C}+9 \mathcal{H}^{2}\left(1-w_{A}\right) \frac{\theta_{A C}}{k^{2}} & =0, \\
\frac{\theta_{A C}^{\prime}}{k^{2}}+\mathcal{H}\left(\frac{a \gamma \bar{\rho}_{B}}{\mathcal{H}\left(1+w_{A}\right)}-2\right) \frac{\theta_{A C}}{k^{2}}-\tilde{\Delta}_{A C} & =0 .
\end{aligned}
$$

These equations can be rewritten in the equivalent form

$$
\begin{aligned}
\tilde{S}_{A C}^{\prime}+\mathcal{H}\left(1+\frac{a \gamma \bar{\rho}_{B}}{\mathcal{H}\left(1+w_{A}\right)}\right) \tilde{S}_{A C} & =\mathcal{H}\left[\frac{5}{3}-w_{A}+\frac{2}{3} \frac{a \gamma \bar{\rho}_{B}}{\mathcal{H}\left(1+w_{A}\right)}=\right] \tilde{\Delta}_{A C}, \\
\tilde{\Delta}_{A C}^{\prime}+\mathcal{H}\left(1-3 w_{A}\right) \tilde{\Delta}_{A C} & =-3 \mathcal{H} \tilde{S}_{A C} .
\end{aligned}
$$

Concerning the sufficiently early Universe, we have $\left|\frac{a \gamma \bar{\rho}_{B}}{\mathcal{H}\left(1+w_{A}\right)}\right| \gg 1$. Under this condition one can obtain the second order equation

$$
\tilde{S}_{A C}^{\prime \prime}+\mathcal{H} \frac{a \gamma \bar{\rho}_{B}}{\mathcal{H}\left(1+w_{A}\right)} \tilde{S}_{A C}^{\prime}+3 \mathcal{H}^{2}\left(1-w_{A}\right) \frac{a \gamma \bar{\rho}_{B}}{\mathcal{H}\left(1+w_{A}\right)} \tilde{S}_{A C}=0
$$

The presence or absence of instabilities is completely determined by the sign of the coupling constant $\gamma$. At negative $\gamma$, there are an anti-damping force and tachyonic instability, which increase indefinitely in the limit $a \rightarrow 0$ and lead to a catastrophic growth of $\tilde{S}_{A C}$ and a non-adiabatic pressure perturbation $P_{\text {nad }}$.

\section{Example 2.}

Another simple coupling is $Q_{A}=-Q_{B}=\gamma \rho_{B}$ [12]. At the radiation dominated stage the background dark sector densities are

$$
\bar{\rho}_{A}=\frac{a \gamma \tau}{3 w_{A}+2} \bar{\rho}_{B}, \quad \bar{\rho}_{B} \propto a^{-3}
$$

This background solution yields

$$
\begin{gathered}
\tilde{E}_{A C} \approx q_{A} \tilde{\Delta}_{B C}, \quad \tilde{E}_{B C} \approx 0 \\
f_{A C}=-\frac{1}{\tau} \frac{3 w_{A}+2}{1+w_{A}} \frac{\theta_{B C}}{k^{2}}, \quad f_{B C} \approx 0,
\end{gathered}
$$


where

$$
q_{A}=\frac{3 w_{A}+2}{3\left(1+w_{A}\right)}
$$

The equations (60)-(63) reduce now to

$$
\begin{aligned}
\tilde{\Delta}_{A C}^{\prime}+3 \mathcal{H}\left(1-w_{A}+q_{A}\left(w_{A}+1\right)\right) \tilde{\Delta}_{A C}+9 \mathcal{H}^{2}\left(1-w_{A}\right)\left(1-q_{A}\right) \frac{\theta_{A C}}{k^{2}} & =3 \mathcal{H} q_{A} \tilde{\Delta}_{B C}, \\
\frac{\theta_{A C}^{\prime}}{k^{2}}+\mathcal{H}\left(6 q_{A}-2\right) \frac{\theta_{A C}}{k^{2}}-\tilde{\Delta}_{A C} & =\frac{1}{\tau} \frac{3 w_{A}+2}{1+w_{A}} \frac{\theta_{B C}}{k^{2}}
\end{aligned}
$$

and

$$
\begin{aligned}
\tilde{\Delta}_{B C}^{\prime}+\theta_{B C} & =0, \\
\frac{\theta_{B C}^{\prime}}{k^{2}}+\mathcal{H} \frac{\theta_{B C}}{k^{2}} & =0 .
\end{aligned}
$$

From the last two equations it follows that one can consistently assume that $\tilde{\Delta}_{B C}=\theta_{B C}=0$, if this condition was fulfilled initially. The first two equations (77) and (78) then give

$$
\begin{aligned}
\tilde{\Delta}_{A C}^{\prime}+3 \mathcal{H}\left(1-w_{A}+q_{A}\left(w_{A}+1\right)\right) \tilde{\Delta}_{A C}+9 \mathcal{H}^{2}\left(1-w_{A}\right)\left(1-q_{A}\right) \frac{\theta_{A C}}{k^{2}} & =0, \\
\frac{\theta_{A C}^{\prime}}{k^{2}}+\mathcal{H}\left(6 q_{A}-2\right) \frac{\theta_{A C}}{k^{2}} & =\tilde{\Delta}_{A C} .
\end{aligned}
$$

Using the definition of $\tilde{S}_{A C}$, the equation (76), and substituting $\mathcal{H}=1 / \tau$, one can write now the second order equation

$$
\tilde{S}_{A C}^{\prime \prime}+\frac{1}{\tau} \frac{11 w_{A}+9}{1+w_{A}} \tilde{S}_{A C}^{\prime}+\frac{2}{\tau^{2}} \frac{11 w_{A}+9}{1+w_{A}} \tilde{S}_{A C}=0 .
$$

Assuming a power-law form of the solution $\tilde{S}_{A C} \propto \tau^{\tilde{n}}$, we obtain the algebraic equation for the power-law index

$$
\tilde{n}^{2}+\frac{10 w_{A}+8}{1+w_{A}} \tilde{n}+2 \frac{11 w_{A}+9}{1+w_{A}}=0
$$

with the roots

$$
\tilde{n}_{ \pm}=-\frac{5 w_{A}+4}{1+w_{A}} \pm \frac{\sqrt{3 w_{A}^{2}-2}}{1+w_{A}} .
$$

The equations (27) and (73) imply that on a large scale $P_{n a d} \approx \frac{\rho_{A}}{\rho} \tilde{S}_{A C} \propto \tau^{3} \tilde{S}_{A C}$. Hence, $P_{n a d}=C_{1} \tau^{n_{+}}+C_{2} \tau^{n_{-}}$, where $C_{1}, C_{2}$ are constants and

$$
n_{ \pm}=-\frac{2 w_{A}+1}{1+w_{A}} \pm \frac{\sqrt{3 w_{A}^{2}-2}}{1+w_{A}} .
$$

The resulting expression agrees with the equation (73) of [12] and a further analysis is identical to the one given in Ref. [12].

\section{CONCLUSION.}

In this paper we considered the evolution of the non-adiabatic perturbations in the models with multiple interacting fluids with $c_{s I}^{2} \neq c_{s I(a d)}^{2}$. We wrote the gauge invariant equations for the variables that determine the non-adiabatic pressure perturbation and the rate of changes of the comoving curvature perturbation $\mathcal{R}$ in the models with energy exchange. The analysis of these equations was made for several particular models. One can see that the quantities $\tilde{S}_{I J}$, introduced in the paper, allow to clarify the features of the non-adiabatic perturbations evolution in multi-fluid cosmological models.

[1] H. Kodama and M. Sasaki, Prog. Theor. Phys. Suppl. 78, 1 (1984). 
[2] A. R. Liddle and D. H. Lyth, Phys. Rep. 231, 1 (1993).

[3] A. D. Linde, Phys. Lett. B158, 375 (1985).

[4] A. A. Starobinsky, JETP Lett. 42, 152 (1985).

[5] L. A. Kofman, A. D. Linde, A. A. Starobinsky, Phys. Lett. B157, 361, (1985).

[6] L. Kofman, A. D. Linde and A. A. Starobinsky, Phys. Rev. Lett. 73, 3195 (1994).

[7] L. Kofman, A. D. Linde and A. A. Starobinsky, Phys. Rev. D56, 3258 (1997).

[8] S. M. Leach, M. Sasaki, D. Wands, and A. R. Liddle, Phys. Rev. D64, 023512 (2001).

[9] L. Boubekeur, P. Creminelli, J. Norena and F. Vernizzi, JCAP 0808, 028 (2008).

[10] C. Gordon and W. Hu, Phys.Rev. D70, 083003 (2004).

[11] K. A. Malik and D. Wands, JCAP 0502, 007 (2005).

[12] J. Valiviita, E. Majerotto and R. Maartens, JCAP 0807, 020 (2008).

[13] J.-H. He, B. Wang, E. Abdalla, Phys. Lett. B671, 139 (2009).

[14] M. B. Gavela, D. Hernandez, L. Lopez Honorez, O. Mena and S. Rigolin, JCAP 0907, 034 (2009).

[15] B. M. Jackson, A. Taylor, A. Berera, Phys. Rev. D79, 043526 (2009).

[16] D. Polarski, A. A. Starobinsky, Nucl. Phys. B385, 623 (1992).

[17] D. Polarski, A. A. Starobinsky, Phys. Rev. D50, 6123 (1994).

[18] C. Gordon, D. Wands, B. A. Bassett and R. Maartens, Phys. Rev. D63 023506 (2001).

[19] J. Hwang, H. Noh, Phys.Lett. B495, 277 (2000).

[20] K. A. Malik, D. Wands and C. Ungarelli, Phys. Rev. D67, 063516 (2003).

[21] G. Ballesteros and J. Lesgourgues, JCAP 1010, 014 (2010).

[22] V. F. Mukhanov, H. A. Feldman and R. H. Brandenberger, Phys. Rep. 215, 203 (1992).

[23] J. M. Bardeen, Phys. Rev. D22, 1882 (1980).

[24] K. A. Malik, arXiv:astro-ph/0101563.

[25] D. Wands, K. A. Malik, D. H. Lyth and A. R. Liddle, Phys. Rev. D62, 043527 (2000).

[26] N. A. Koshelev, arXiv:0912.0120 [gr-qc]. 